\title{
Enhance User Engagement using Gamified Internet of Things
}

\author{
Ruowei Xiao \\ Tampere University \\ ruowei.xiao@tuni.fi
}

\author{
Zhanwei Wu \\ Shanghai Jiao Tong University \\ zhanwei_wu@sjtu.edu.cn
}

\author{
Oguz Turan Buruk \\ Tampere University \\ oguz.buruk@tuni.fi
}

\author{
Juho Hamari \\ Tampere University \\ juho.hamari@tuni.fi
}

\begin{abstract}
Gamification is considered as a promising approach to enhance people's engagement in many social or technical systems, thus is supposed to play an important role in future Internet of Everything (IoE). Although gamification elements have already been reported in various IoT research, there still lacks answers about how gamification may affect user engagement in IoT systems and through what paths. In present work, we are synthesizing and analyzing existing research efforts in these emerging fields to provide implications for future IoE development. The results are categorized into three dimensions by considering cognitive-behavioral outcome, procedural stage and population scale.
\end{abstract}

\section{Introduction}

The Internet of Things (IoT) is further evolving into the Internet of People (IoP) [1] and the Internet of Everything (IoE) [2]. The profound role of people as contributors of data, intelligence and other potential value, has been emphasized. Rather than sheer technical problems, many common targets of current IoT systems, such as sustainability, public health and urban smartness etc., are complicated socio-technical challenges unable to be settled without active involvement of users and collective wisdom generated from the co-creation and co-innovation progress. As a result, user engagement has become one of the vital design and development goals of current IoT applications and services.

Among others, gamification presents itself a de facto approach for increasing user engagement in various application domains such as health [3], education [4], governance [5], marketing [6, 7] and others, by transforming systems and services to afford gameful experience [8, 9]. Also among recent trends of IoT, an increasing interest in combining IoT and gamification has newly emerged, explicitly in the fields such as education, crowdsourcing, health, smart cities etc., where complicated human factors and multiple stakeholders are typically involved [10].

In this article, we have reviewed the literature body in the emerging field of gamified IoT applications. Search query was based on keywords combining both IoT and gamification, and results were retrieved from the Scopus engine, as it indexes all other potentially relevant databases, e.g. ACM, IEEE, Springer. In total, 196 hits were acquired at the time of October 2019, among which we selected empirical studies with evaluation results from conference papers, articles and book chapters. Exemplar usages of gamification elements under IoT context in each study were analysed qualitatively, and synergized effects were discussed from three different perspectives of user engagement, respectively: 1) Cognitive-behavioral outcome; 2) Procedural stage; And 3) Population scale. By extracting and structurally mapping particular gamified IoT approaches towards engagement outcomes, this article intends to define a well-structured, user-centered design space, and generate pragmatic design implications and insights for the future design and development of more engaging IoT systems.

The rest of this paper will be structured as follows: Section 2 will provide an overview of engagement and its related aspects, based on which representative projects and studies will further be categorized and analyzed in Section 3, with specific emphasis on the usages of gamified elements. Finally, in Section 4 particular gamified elements and their respective design implications will be extracted and concluded.

\section{User Engagement: Cognitive- Behavioral Outcome, Procedural Stage and Population Scale}

What is "engagement"? To answer the question, we firstly reviewed the most widely acknowledged definitions of engagement in diverse application domains. For example, a definition from information system is that "a quality of user experiences with technology" [11] that emerged as a consistent process 


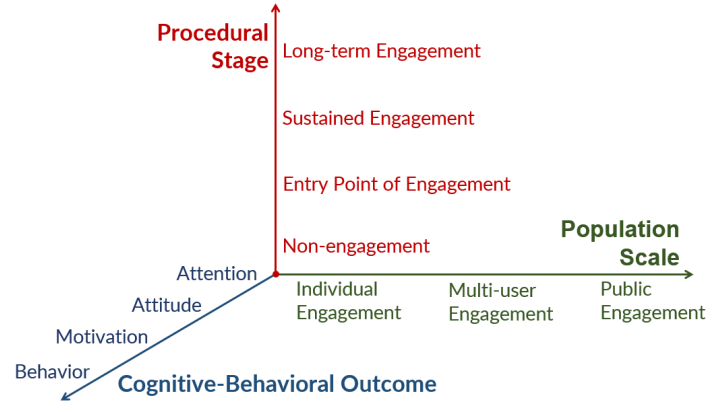

Figure 1. Engagement Outcome Dimensions for Categorizing Gamified IoT Systems

with distinguishable attributes inherent at each stage in that process, e.g. the point of engagement, period of engagement, disengagement, reengagement. In business domain, engagement is defined as "a psychological state that appears from an important thing (e.g. a brand) due to interactional experiences and creative participation" [12]. Education research emphasizes more on the different psychological mechanisms of engagement: cognitive engagement, emotional engagement and behavioral engagement [13]. While public governance domain considers engagement as "actions" that "citizens take in order to pursue common concerns and address problems in the communities they belong to" [14].

The divergence makes it difficult to reach a consensus in defining what is engagement across different disciplines. However, there does exist certain aspects or facets that commonly attract attentions from research community, among which we identified three distinctive dimensions: 1) Psychological-behavioral outcome; 2) Procedural stage; And 3) Population scale. We categorized related studies and analyzed respective impacts of gamified elements according to these three dimensions, not only because they are among the most common perspectives emerged from current literature, but also they facilitate the pragmatic design and development of gamified IoT systems by clarifying expected engagement outcomes more precisely.

Cognitive-Behavioral Outcome: Mainstream research in all related fields agree that engagement has impacts on users' behavior through a relatively complicated cognitive-behavioral process, although the underlying mechanisms are not completely clear yet. Educational researchers have done the most in-depth research on this issue and recommend to study engagement from cognitive, emotional and behavioral factors [12]: 1) Cognitive engagement involves the psychological investment of a person in the process of engaging the target, which is usually related to paying/regulating one's attention [15]; 2) Emotional engagement involves affective and emotional arousal while engaging the target, which (together with cognitive engagement) can affect one's attitude [16] and motivation/intention [17]; 3) Behavioral engagement involves the participation and involvement in actual behavior. Previous research recognized the correlation of attention, attitude, motivation and behavior in engagement process as a continuum of cognitive-behavioral states $[18,19]$. And rather than anchored at one single state, engagement was regarded as a consistent, progressive process where transitions among different states were frequently observed $[12,16]$. For instance, a complete progress of engagement may enter at the attention state, while exit at attitude or behavior state.

Procedural Stage: Independent from its impact on cognitive-behavioral outcomes, engagement itself emerged as "a process with distinguishable attributes inherent at each stage in that process" [11]. In this article, the procedural stages of engagement are defined as: 1) Non-engagement; 2) Entry Point of Engagement; 3) Sustained Engagement; and 4) Long-term Engagement. We argue that it is of practical significance to discuss these procedural stages separately, as the particularity of each stage may entail different system designs and approaches. To better illustrate, Entry point of engagement is where users' attentions are first captured and engagement is initialized. As the procedure continues and users do not drop out from the target experience, it will possibly extend to the phase of sustained engagement, which usually takes place in non-transient, sequential behaviors that consist of more than one atomic action. Though maybe consisting of multiple dynamic cycles of engagement-disengagement-reengagement behaviors, the long-term engagement actually reflects a stable retention of engagement in the long run. On the contrary, if users' interests and motivations are not persistently maintained, it is likely for their state move towards non-engagement, the process of which can also be defined as "disengagement". Similarly, if users feel that their goal has been achieved or needs fulfilled, it is also possible for them to break away from their current engaged state. It is also noteworthy that different stages may be involved and dynamically occur/reoccur during the overall procedure of user engagement.

Population Scale: According to how large scale of expected users is to get involved in the same target outcome simultaneously, user engagement can be generally categorized into: 1) Individual engagement, referring to the kind of engagement, whose target outcome can be achieved by engaging individual user, either with or without other presences, even 
if massive users can actually be present in single application scenario at the same time; 2) Multi-user engagement, different from individual engagement, requires more than one participant and/or stakeholders to be engaged in order to achieve a collective goal or group behaviors, which possibly ranging from family-level to community-level engagement. 3) Public engagement, referring to crowd/public level of engagement targeting unspecified user groups or the general public.

In the coming section, the aforementioned three dimensions will be used for qualitatively analyzing gamified IoT applications, which is supposed to facilitate accurate gamification of IoT systems to achieve specific engagement outcomes.

\section{Qualitative Analysis: Exemplar Gamified IoT Systems and their Engagement Outcomes}

\subsection{Cognitive-Behavioral Outcome}

As suggested in the previous section, engagement can be regarded as a consistent progress featured by intervals of different cognitive-behavior states. We will concentrate on presenting analysis results of [Attention, Attitude] and [Motivation, Behavior], mainly because: First, engagement outcomes that cover longer cognitive-behavior ranges like [Attention, Behavior] can be broken down into smaller intervals; And second, most of the reviewed studies were found exit at either attitude or behavior, while both attention and motivation appeared to be intermediary phases.

[Attention, Attitude]. Our analysis showed that knowledge impartation and storytelling were generally considered two effective gamification strategies to foster attitudinal transition in IoT applications. According to the elaboration likelihood model theory (ELM) [20], the former belongs to the central route towards attitude changing, while the latter belongs to the peripheral route. An example of storytelling was provided in [21], where an escape-room-style of pervasive game was designed to raise public cautiousness about the potential abuse of AI-enhanced surveillance networks in modern smart cities. During the escape progress, players were asked to experience AI-integrated face recognition and hunt surveillance cameras in the actual urban area. By connecting to users' own experience and feeling via critical storytelling and a dystopia plot, the authors claimed to successfully sensitize people about existing surveillance infrastructure according to their pre-post questionnaires. On the other hand, imparting knowledge, though may be manifested in various forms like quizzes [22], instructional NPC [23], informative feedback [24] etc., intends to change users' attitudes by educating and instilling them with information and knowledge. In [25], a serious game was built to promote privacy protection among smart watch users. By simulating a smart-watch-like interface, the game allowed players to be challenged by a variety of privacy threats, learn necessary methods to counter the threats as well as hands-on practice of security configuration. In their pretest-posttest evaluation, imparting knowledge about privacy risks was proved to be significantly effective in increasing users' concerns regarding specific privacy aspects. Also, compared with traditional instruction, gamified/gameful impartation can provide contextualized, interactive learning contents and was reported to contribute to self-efficacy and a longer retention of knowledge [26]. However, the research also identified an interesting gap between users' attitudes and behaviors, supported by both their high concerns about location tracking and meanwhile low adoption of disabling GPS functions on their watches.

[Motivation, Behavior]. Psychology research showed that attitude-behavior gaps may widely exist [27], indicating that attitude is a prerequisite, however not sufficient phase in order to obtain a certain behavior outcome. Even if people are attituded, there must be enough incentives to motivate them to act, which explains that the motivation phase usually appears as a transitional, but very important entering stage for the behavior state. According to Self-Determination Theory [28], motivation generally derives from either extrinsic incentives, or intrinsic incentives. Both are important stimuli towards actual "conversion rate" of user behaviors. Frequently used extrinsic incentives include monetary rewards [29], and other material rewards like in [30], in which bicycles, subscriptions to eco-friendly mobility services and other similar rewards were given to the users of a gamified itinerary planning application, who outperformed in achieving its sustainable mobility tasks and goals. While intrinsic incentives usually stem from the design of gameful experience itself. [31] utilized a wearable sensor based pervasive game to monitor a player's daily physical and social activeness, the data of which were reflected by his/her game avatar's attributes e.g. strength, dexterity, charisma etc. Although the attributes were not directly related to rewards, the stronger attributes, the more likely for players to obtain boost items in other related pervasive social games that in turn stimulated participants to be more actively involved in physical and social activities. Both examples here showcased that target behaviors can be designed as part of, or extension 
of the incentives, thus a positive behavioral cycle can be established and further reinforced.

To conclude this subsection, attitudinal and behavioral transition are found to be two major cognitive-behavioral outcomes of user engagement in current literature. Knowledge impartation and storytelling were commonly adopted strategies to change users' attitudes, and extrinsic incentives as well as intrinsic incentives to change behaviors. While conventional usage of some gamification approaches like extrinsic incentives appeared to be similar when compared with other fields, however, technical affordance introduced by IoT such as ubiquitous sensing, also brought unique design trend of blurring the boundary between physical and cyber realities, thus engaging people in a more precise and profound way.

\subsection{Procedural Stage}

According to different procedural features, the engagement process can be further divided into four stages. And in some gamified IoT applications, certain stages were found accentuated more than the others, contributing to different design and deployment of gamification elements and strategies.

Entry Point of Engagement. Crowdsensing, or participatory sensing, is an emerging application domain of crowdsourcing based on mobile sensing technology. As the contribution of sensed data relies on user participation, this kind of application usually concentrates more on directing potential data contributors towards the entry point of engagement rather than retaining a steady returning usage. [32] proposed a gamified AR mobile application to collect and tag flood data for environmental monitoring purposes. When detected nearby a predefined point of interest (POI), vibration and automatic switch-on of mobile camera were used to raise a player's attention, even if he/she was not using the application. The player was then guided to face specific orientation and capture a "little animal" in an AR view by taking pictures that included information of interest.

Sustained Engagement. While the entry point of engagement is closely associated with acquiring users' attention and interests, it may entail a more sophisticated mechanism from an application or service to maintain users' motivation so that the progress is prolonged into sustained engagement. Telerehabilitation systems are among such applications as they usually require intensive and continuous physical and/or cognitive exertion from the patients when using the systems. [33] proposed a gamified telerehabilitation exergame, where self-adaptive challenges were deployed to automatically moderate game difficulty levels according to the patient's real-time performance and EEG-detected stress level. Meanwhile, a vibrotactile actuator provided hints to the patient by haptic feedback, when he/she encountered difficulties during the session. Thus, patients were prevented from dropping out of rehabilitation. Examples were also found in smart cultural tourism. Aiming at routing visitors between a set of 8 differently-themed museums across the Dutch-German borders, [34] integrated a modular, loose-coupled system architecture. It allowed flexible, non-linear assembly of various game components and story plots, which were supposed to guide visitors' awareness towards the next point of interests, and motivate them to continue inter-location visits without restricting their visiting sequence; Meanwhile, location-sensitive, content-specific mini-games, e.g. riddles, cultural caching, AR beat-them-up etc., were used to enrich visitors' intra-location experience.

Long-term Engagement. More frequently, long-term user engagement is desirable because it lands on a stable retention of target outcomes, thus ultimately helping shaping new cognitive-behavioral patterns or even values and beliefs of people. [35] brought up a social gaming platform based on in-vehicle sensor networks to promote long-term safe driving behaviors. Realtime driver performance evaluation was conducted based on vehicle sensor data, and drivers competed with their contacts via social network function. Similarly, [30] tried to encourage voluntary transitions in citizens' travel patterns for a sustainable mobility purpose. A gamified itinerary planning mobile application was provided to users, allowing tracing users' mobility related behaviors and delivery of gamified features. Among all the gamified elements provided, social features and contents update like limited time challenges, weekly and final leaderboards etc., were identified as the key elements to "encourage user engagement and retainment" throughout the game's duration. By conducting a 9-week field test with 110 active participants, the researchers evaluated the gamification efficacy and reported positive results in supporting long-term citizen engagement and forming new mobility habits.

Non-engagement. On one hand, the wide usage of social gaming features for long-term user engagement is aligned with the general finding in other research areas [36]; On the other hand, In further support of this conclusion, excessive social competition was found as a source of demotivation and trigger user disengagement as reported in [37]. Therefore, competition-based gamified elements such as leaderboards must be carefully designed to "appeal to latecomers who 
enter the game at any point", and the effectiveness must be independent from the accumulation of game status in consideration of long-term engagement [30]. Another interesting finding of non-engagement was that even identical gamified incentives might lead to entirely contrary results. Similar to [30], [38] was also targeted on behavior shift of citizen mobility, and an alike gamified mobile application involving limited time challenges and social networking functions was developed to promote long-term sustainable travel patterns. However, it was reported that only recruited participants started using the application only during the period when an experiment was carried out. The researchers admitted that despite promotional activities, the application itself was not commercially successful and unable to obtain a city-wide acceptance. Moreover, in [39] an energy generating device was built to supply electrical energy to environmental sensors while engaging the public in a game. The device was installed in the public space of a college campus, with a case box decorated with animation characters and a physical crank for passengers to wind. The system logs showed that the device managed to attract passengers' interests only within the beginning ten minutes of each interaction, and the total interaction amount also drastically dropped after the first 3 months after the device's installation. It scaffolded the fact that an application may succeed in engaging users at a certain procedural stage, e.g. entry point of the engagement, while failing to extend the engagement to another stage, e.g. sustained or long-term engagement, if without properly targeted strategy.

In this subsection, different approaches were identified according to the procedural stages of engagement. At the entry point of engagement, sensory stimulus like vibration or other multisensory interaction was considered effective to obtain users' initial attention and interests. In sustained engagement, interactability, including but not limited to elements such as contextual awareness, self-adaptive challenges, informative feedback and modular game components etc., was the key to timely, situational as well as appropriate user interactions for keeping users engaged. While in long-term engagement, both social interaction and contents update were two specific approaches closely associated with steady user engagement in the long run. We also discussed non-engagement, however, a scarcity in literature indicates that this issue has not yet obtained enough attention from research community, which is possibly due to the publication bias [40]. Yet, our study suggests that analysis of disengagement (what parts of the approaches lead to abandonment of the application) with Gamified IoT is an under-explored space in the field.

\subsection{Population Scale}

Our analysis found that the target user scale to be engaged simultaneously would also affect the gamification strategies and their corresponding performance. We argue that it is due to the fact that the patterns of people-people and people-system interactions vary significantly as the population scale escalates.

Individual Engagement. Aside from common gamified elements such as points, badges [37, 41, 42] etc., in gamified IoT systems we also witnessed a unique tendency to utilize IoT extended sensing ability to provide dynamically generative, highly personalized gamified contents to achieve individual engagement. In [37], players were encouraged to associate their personal health data collected from wearable devices to an "endless-runner" style game. Instead of direct reward of scores or points, in-game terrains and obstacles were constructed based on the total length of the user's active and sedate duration in the previous day respectively. A similar mechanism was also adopted by [43]. Projection was another frequently emphasized mechanism in individual engagement, which usually featured with customizable avatars, personification etc. Different from its conventional counterpart in traditional gamified applications that are predefined or manually configured, automatic projection enhanced by IoT sensing allows data-driven, automatic mapping between project subjects and their "digital twins". In [42], soil moisture and sunlight sensors were adopted for monitoring the stress level of house plants. The collected data was personified into cartoon characters in a mobile game for reminding players to take care of their plants in a real time manner. Their user experiment reported positive emotional involvement when interacting with the personified plant. Similarly, in [44] a virtual tree was used for projecting occupants' energy consumption data in public buildings. We argue that automatic projection is not only able to generate more immersive experience and meaningful data representation, but also easier to get users emotionally associated with the target contents via vivid reflections of either players themselves or other subjects. To specifically note that, most of the aforementioned studies also involved social features like social feed, social network sharing or leaderboard [37, 41, 42]. However, they were tagged as individual engagement due to the reason that although a massive number of users can actually be present at the same scenario at the same time, the target behavior can essentially be 
achieved by either with or without other users' presence. Multi-user engagement. Multi-user engagement usually requires for more than one participant and/or stakeholders to be engaged in order to achieve a collective goal or group behaviors. Examples included teachers and students at the same education field [45, 46], therapists and patients using same telerehabilitation system [47], as fostering connection and communication among multiple participants is usually also among the goals of user engagement. Different patterns of in-game social interactions were enhanced or mediated by IoT technical affordance, which could be defined as IoT-mediated social interaction: 1) IoT-mediated Competition: [48] tried to detect and monitor healthcare workers' compliance of hand hygiene behaviors, by launching a BLE-beacon-based proximity sensor network in an ICU ward. Public leaderboard and real-time ranking were reported to have raised nurses' motivations to compete with their colleagues at the same workplace, thus contributing to both the self-awareness of health workers and the entire compliance rate of hand hygiene behaviors; 2) IoT-mediated Collaboration: Collaboration was the social interaction pattern that most commonly observed in intra-family engagement. For instance, [49] proposed a savings management system for promoting children's savings behaviors, which consisted of an IoT-enhanced piggy bank device and a mobile application on top. Together parents and children were able to establish savings goals, release requests (e.g. chores), and set up the rewards for successfully accomplishing the goals and requests, etc. The authors claimed that the process not only helped children to cultivate a sustainable financial concept and behavior, but also "strengthen the family bond". Similar mechanism was also adopted by [50], where a rule-based home automation platform was gamified to facilitate collaboration among family members, by exchanging requests for creating rules to control smart home appliances; 3) IoT-mediated Collaboration-based Competition: Aiming at reducing occupants' energy wastage, [44] deployed low-cost IoT devices (NFC/BLE), smart meters and plugs to monitor energy consumption of public buildings. And a serious mobile game was developed to engage and motivate users to involve in the team competition of energy saving knowledge and behaviors. Collaboration-based competitive socialization was given specific emphasis because most employees believed that "only team efforts would be effective towards energy conservation at work". Another example was found in the field of edutainment robotics. A robot race game combining RFID/NFC technology was described in [51], where the speed of robot reaching the finish line depended on the learning performance of each team. The efficacy of the proposed system was supported by control group user experiments. Compared with conventional lecture based pedagogy, the gamified IoT approach was reported no difference in learning performance, but also with a more "fun and socializing" perception of learning process from students.

Public engagement. Generally, public engagement target on unspecified user groups at a crowd/public level. Most crowdsensing related applications belong to this genre, as well as public smart services that seek for positive behavior changes at a societal level. While the introduction of IoT gives access to new technical affordance and design possibilities, it also places extra technical barriers upon users. Therefore, if gamified IoT systems attempt to engage as wide audience as possible, system accessibility, referring to a broader inclusion of cost and/or time affordability, data achievability, and technical reachability etc., becomes a prerequisite and actually determines to what depth and farth that the targeted public can be reached. [30] tried to address a public health issue that many smart health products/devices were beyond the reach of the population really in need, by providing publicly-accessible smart cycling machines with some gamified reward. Moreover, in the public health campaign in Singarpore [52], around 3000 RFID tags were disseminated to the citizens who took part in a walking promotion program. By checking in at the designated checkpoints using the tag, participants were able to collect points to win rewards. As about $65 \%$ of the participants were aged 50 and above, the authors claimed that the program demonstrated how merged solution of gamification and IoT technology "can appeal to less tech-savvy groups and influence their behaviors." The scale of public engagement in most occasions, also entails profound support from other aspects like administration, finance and policy, which, however, are considered beyond the scope of this paper.

To briefly summarize, it can be observed in current literature that a few system attributes appear to be associated with specific engagement scales. Explicitly, personalization (e.g. personalized generative contents) and aesthetic appealing (e.g. automatic projection) are emphasized in individual engagement; As the target user scale increases, the sociability (e.g. IoT-mediated social interaction) of the system becomes predominant for actively engaging multiple users; And when targeting unspecified massive public, the overall accessibility (e.g. hardware accessibility, data accessibility, technical reachability etc.) of the systems and services is considered of specific significance. 


\section{Discussion and Conclusion}

In this section, we will discuss particular gamified system elements, their efficacy regarding specific engagement targets, as well as respective technologies and design implications. Due to the space limit, some listed studies were not previously mentioned. Instead they are clustered and concluded in the Table 1 as similar usage of gamified elements were identified. Also, other than covering all the minor usages of each element, Table 1 contains only the most prominent findings from our review.

Sensory Stimulus, frequently referring to interactions that appeal to or utilize one or more senses of users, e.g. visual, auditory, tactile, olfactory, gustatory stimulus. It is generally coupled with the technical affordances of IoT, and appears to be particularly effective in attracting users' attention and interests at the start point of engagement. Another frequent usage of sensory stimulus in gamified IoT systems is to provide real-time informative feedback for retaining users' engagement.

Knowledge Impartation belongs to the central route towards attitude changing according to the ELM theory [20]. However, it remains to be investigated the relationship with disengagement as well. In more occasions, the two elements of storytelling and knowledge impartation can be possibly combined to provide a stronger persuasion effect like in $[43,57]$.

Storytelling, on the other hand, belongs to the peripheral route towards attitude changing, and helps get users associated emotionally [20]. The construction of narrative can help retain attention and re-engage users as shown in [34].

Projection, in this paper, is used for describing the mapping mechanism towards other, usually animate manifestations, from either the user (e.g. Avatar etc.), or non-user subjects (e.g. NPC, personification of data etc.). The former usage contributes to increasing overall system customizability [45, 23, 25], while the latter is usually associated with informative feedback $[42,25,54]$ and metaphorical representation of abstract data [44]. Projection helps nudge users into desirable attitude or behavioral transitions by creating emotional relatedness [59].

Simulation, though not previously discussed, refers to transporting users to an artificial environment to generate the sense of immersion. Technologies like VR/AR are tightly coupled with the simulation element to create circumstances allowing for users' behavior training. It is specifically preferred due to a lower cost, better system accessibility, and extensive applications unable to be achieved in a real environment.

Intrinsic Incentive, stems from the internal mechanisms and elements of the systems, e.g. challenges, achievements, badges, goals etc. A promising design implication manifested in current literature is to provide more personalized, individualoriented intrinsic incentive according by collecting and analyzing user preference data, as suggested in [30].

Extrinsic Incentive, on the contrary to intrinsic incentive, derives from outside the systems, e.g. monetary or material rewards. It has been widely applied in attracting public attention and large-scale engagement. A well-designed reward itself can actually contribute to a positive cognitive-behavioral cycle and hence reinforce the targeted engagement outcomes [30]. Social Interaction, including but not limited to direct (e.g. competitions, collaborations, collaboration based team competitions etc.) or indirect (e.g. social feeds and sharing etc.) social contacts with others. As previously discussed, excessive competitive elements may result in users' being discouraged and thus disengaging. Moreover, we have also noticed that most current gamified IoT applications utilize either sheer "physical" or "virtual" social interaction, which implies a less exploited design space that can be extended by blending both online and offline users together. The hybrid player interactions, which is firstly derived from the genre of pervasive games [60] and leverages ubiquitous information of IoT, can further associate wider target users via natural social connections and enriched social experience as proposed in [58].

Contents Update, also known as contents novelty. A system regularly updates its contents to maintain and retain its users' curiosity and interests. Many adoptable mechanisms including limited time challenges/tasks, weekly/monthly ranking/leaderboard, download contents (DLC), patches etc., can actually be found in current Massively Multi-player Online (MMO) games. Aside from long-term engagement, content update is also considered effective in directing users' focus towards situational topics and themes, as well as refreshing accumulated game status hence appealing to potential novice users to the system. However, it also indicates the necessity of a platform for content maintenance and update.

Conclusion. With the role of user participation being more and more centralized, modern smart systems and services have particularly enlisted engagement as a common goal. However, the meta-construct nature of engagement entails that different design strategies must be adopted accordingly. To this end, our review showed that gamification is a potently engaging approach that can be embedded coherently into the mechanics, dynamics and aesthetics design of IoT applications. Reversely, the technical affordances of IoT also bring 


\begin{tabular}{|l|l|l|l|l|}
\hline Gamified Elements & Engagement Stage & Engagement Scale & Cognitive-Behavioral Phase & Reference \\
\hline \hline Sensory Stimulus & Entry point, sustained & Individual & $\begin{array}{l}\text { Attention, attitude, motivation, } \\
\text { behavior }\end{array}$ & {$[32,33,53]$} \\
\hline $\begin{array}{l}\text { Knowledge } \\
\text { Impartation }\end{array}$ & $\begin{array}{l}\text { Sustained, } \\
\text { non-engagement }\end{array}$ & Attitude, motivation, behavior & {$[22,23]$} \\
\hline Storytelling & Entry point, sustained & Individual & $\begin{array}{l}\text { Attention, attitude, motivation, } \\
\text { behavior }\end{array}$ & {$[21,34]$} \\
\hline Projection & Sustained & Individual & Motivation, behavior & {$[42,45,44,54]$} \\
\hline Simulation & Sustained & Individual, multi-user & Motivation, behavior & {$[54,55,56]$} \\
\hline Intrinsic Incentive & Sustained, long-term & Individual, multi-user, public & Motivation, behavior & {$[41,23,31,57]$} \\
\hline Extrinsic Incentive & Entry point, sustained, long-term & Individual, multi-user, public & $\begin{array}{l}\text { Attention, attitude, motivation, } \\
\text { behavior }\end{array}$ & {$[30,38,49,52,29]$} \\
\hline Social Interaction & $\begin{array}{l}\text { Sustained, } \\
\text { non-engagement }\end{array}$ & Motivation, behavior & $\begin{array}{l}{[37,41,42,48,49,} \\
50,44,51,58]\end{array}$ \\
\hline Contents Update & Entry point, long-term & Multi-user, public & Individual, multi-user, public & Motivation, behavior \\
\hline
\end{tabular}

Table 1. Gamification Elements and Corresponding User Engagement Performance

novel possibilities and changes towards gamification, or in a broader sense, playful user experience design. Future research in this direction may emphasize on leveraging IoT from the following aspects: 1) Increased overall interactability; 2) Ubiquitous context awareness; And 3) Enhanced interfaces between physical (reality) and digital (virtual) activities. We also identified a scarcity in the research regarding users' disengagement behavior and the causal factors, which will be an interesting topic worthy of future investigation.

From a more pragmatic perspective, the implication of this article will facilitate industry practitioners and service providers to engage their target users more precisely and effectively, aiming at a variety of population scales, cognitive-behavioral outcomes and application domains. On the other hand, the enhanced user engagement reinforces the proactive roles of uses, as both data consumers and contributors, fueling extensive service smartness, e.g. precise customer profiling, situational context awareness, etc., which is believed to constitute the foundation of the future full-fledged IoP [1].

Limitation. Although many efforts of this research has been made to clarify the correlation between specific gamification approaches and the resulting engagement performance, some of our findings may need further validation since many studies actually adopted gamified multiple elements and methods. As a result, the intertwined effects and lacking of control variables based evaluations made it challenging to identify independent variables and significant differences among driving factors. Therefore, further in-depth and systematic investigation must be made in this area.

Future work. Our future study will be focused on a few system attributes, e.g. accessibility, interactability, sociability etc., that emerged from this literature review and appeared to be the common "handlers" of both IoT technology and gamification for enhancing user engagement, in order to further investigate the synergized effects of IoT and gamification.

\section{References}

[1] J. Miranda, N. Mäkitalo, J. Garcia-Alonso, J. Berrocal, T. Mikkonen, C. Canal, and J. M. Murillo, "From the internet of things to the internet of people," IEEE Internet Computing, vol. 19, no. 2, pp. 40-47, 2015.

[2] M. H. Miraz, M. Ali, P. S. Excell, and R. Picking, "A review on internet of things (iot), internet of everything (ioe) and internet of nano things (iont)," in 2015 Internet Technologies and Applications (ITA), pp. 219-224, IEEE, 2015.

[3] L. Hassan, A. Dias, and J. Hamari, "How motivational feedback increases user's benefits and continued use: A study on gamification, quantified-self and social networking," International Journal of Information Management, vol. 46, pp. 151-162, 2019.

[4] M. D. Hanus and J. Fox, "Assessing the effects of gamification in the classroom: A longitudinal study on intrinsic motivation, social comparison, satisfaction, effort, and academic performance," Computers \& education, vol. 80, pp. 152-161, 2015.

[5] L. Hassan and J. Hamari, "Gameful civic engagement: A review of the literature on gamification of e-participation," Government Information Quarterly, p. $101461,2020$.

[6] C. F. Hofacker, K. De Ruyter, N. H. Lurie, P. Manchanda, and J. Donaldson, "Gamification and mobile marketing effectiveness," Journal of Interactive Marketing, vol. 34, pp. 25-36, 2016.

[7] N. Xi and J. Hamari, "Does gamification affect brand engagement and equity? a study in online brand communities," Journal of Business Research, vol. 109, pp. 449-460, 2020.

[8] J. Hamari, "Gamification," The Blackwell Encyclopedia of Sociology, pp. 1-3, 2007.

[9] S. Deterding, D. Dixon, R. Khaled, and L. Nacke, "From game design elements to gamefulness: defining" gamification"," in Proceedings of the 15th international academic MindTrek conference: Envisioning future media environments, pp. 9-15, 2011.

[10] A. Kankanhalli, Y. Charalabidis, and S. Mellouli, "Iot and ai for smart government: A research agenda," 2019.

[11] H. L. O'Brien and E. G. Toms, "What is user engagement? a conceptual framework for defining user engagement with technology," Journal of the American society for Information Science and Technology, vol. 59, no. 6, pp. 938-955, 2008.

[12] R. J. Brodie, L. D. Hollebeek, B. Jurić, and A. Ilić, "Customer engagement: Conceptual domain, 
fundamental propositions, and implications for research," Journal of service research, vol. 14, no. 3, pp. 252-271, 2011.

[13] S. L. Christenson, A. L. Reschly, and C. Wylie, Handbook of research on student engagement. Springer Science \& Business Media, 2012.

[14] C. Zukin, S. Keeter, M. Andolina, K. Jenkins, and M. X. D. Carpini, A new engagement?: Political participation, civic life, and the changing American citizen. Oxford University Press, 2006.

[15] M. Schmidt, V. Benzing, and M. Kamer, "Classroom-based physical activity breaks and children's attention: cognitive engagement works!," Frontiers in psychology, vol. 7, p. 1474, 2016.

[16] F. J. Heide, N. Porter, and P. K. Saito, "Do you hear the people sing? musical theatre and attitude change.," Psychology of Aesthetics, Creativity, and the Arts, vol. 6, no. 3, p. 224, 2012.

[17] A. J. Martin, "Part ii commentary: Motivation and engagement: Conceptual, operational, and empirical clarity," in Handbook of research on student engagement, pp. 303-311, Springer, 2012.

[18] Y. Li and R. M. Lerner, "Interrelations of behavioral, emotional, and cognitive school engagement in high school students," Journal of Youth and Adolescence, vol. 42, no. 1, pp. 20-32, 2013.

[19] J. A. Fredricks, P. C. Blumenfeld, and A. H. Paris, "School engagement: Potential of the concept, state of the evidence," Review of educational research, vol. 74 , no. 1, pp. 59-109, 2004.

[20] R. E. Petty and J. T. Cacioppo, Communication and persuasion: Central and peripheral routes to attitude change. Springer Science \& Business Media, 2012.

[21] T. Kihara, R. Bendor, and D. Lomas, "Designing an escape room in the city for public engagement with ai-enhanced surveillance," in Extended Abstracts of the 2019 CHI Conference on Human Factors in Computing Systems, pp. 1-6, 2019.

[22] G. Mylonas, D. Amaxilatis, L. Pocero, I. Markelis, J. Hofstaetter, and P. Koulouris, "An educational iot lab kit and tools for energy awareness in european schools," International Journal of Child-Computer Interaction, vol. 20, pp. 43-53, 2019.

[23] M. Casals, M. Gangolells, M. Macarulla, A. Fuertes, V. Vimont, and L. M. Pinho, "A serious game enhancing social tenants' behavioral change towards energy efficiency," in 2017 Global Internet of Things Summit (GIoTS), pp. 1-6, IEEE, 2017.

[24] A. C. Amaro and L. Oliveira, "Iot for playful intergenerational learning about cultural heritage: The locus approach.," in ICT4AWE, pp. 282-288, 2019.

[25] M. Williams, J. R. Nurse, and S. Creese, "(smart) watch out! encouraging privacy-protective behavior through interactive games," International Journal of Human-Computer Studies, vol. 132, pp. 121-137, 2019.

[26] P. Wouters, C. Van Nimwegen, H. Van Oostendorp, and E. D. Van Der Spek, "A meta-analysis of the cognitive and motivational effects of serious games.," Journal of educational psychology, vol. 105, no. 2, p. 249, 2013.

[27] R. H. Fazio and D. Roskos-Ewoldsen, "Acting as we feel," Persuasion: Psychological insights and perspectives, pp. 281-303, 2005.
[28] R. M. Ryan and E. L. Deci, "Self-determination theory and the facilitation of intrinsic motivation, social development, and well-being.," American psychologist, vol. 55, no. 1, p. 68, 2000.

[29] A. G. Briones, P. Chamoso, A. Rivas, S. Rodríguez, F. De La Prieta, J. Prieto, and J. M. Corchado, "Use of gamification techniques to encourage garbage recycling. a smart city approach," in International Conference on Knowledge Management in Organizations, pp. 674-685, Springer, 2018.

[30] R. Kazhamiakin, A. Marconi, A. Martinelli, M. Pistore, and G. Valetto, "A gamification framework for the long-term engagement of smart citizens," in 2016 IEEE International Smart Cities Conference (ISC2), pp. 1-7, IEEE, 2016.

[31] I. Hwang, Y. Lee, T. Park, and J. Song, "Toward a mobile platform for pervasive games," in Proceedings of the first ACM international workshop on Mobile gaming, pp. 19-24, 2012.

[32] M. Krommyda, E. Sdongos, S. Tamascelli, A. Tsertou, G. Latsa, and A. Amditis, "Towards citizen-powered cyberworlds for environmental monitoring," in 2018 International Conference on Cyberworlds $(\mathrm{CW})$, pp. 454-457, IEEE, 2018.

[33] M. Oliver, M. A. Teruel, J. P. Molina, D. Romero-Ayuso, and P. González, "Ambient intelligence environment for home cognitive telerehabilitation," Sensors, vol. 18, no. 11, p. 3671, 2018.

[34] N. Fischöder, I. A. Iurgel, T. I. Sezen, and K. van Turnhout, "A storytelling smart-city approach to further cross-regional tourism," in Interactivity, Game Creation, Design, Learning, and Innovation, pp. 266-275, Springer, 2018.

[35] P. Brauner and M. Ziefle, "Exergames for elderly in ambient assisted living environments," in International Internet of Things Summit, pp. 145-150, Springer, 2014.

[36] M. Zhang, L. Guo, M. Hu, and W. Liu, "Influence of customer engagement with company social networks on stickiness: Mediating effect of customer value creation," International Journal of Information Management, vol. 37, no. 3, pp. 229-240, 2017.

[37] R. Gawley, C. Morrow, H. Chan, and R. Lindsay, "Bitrun: Gamification of health data from fitbit ${ }^{\circledR}$ activity trackers," in International Conference on IoT Technologies for HealthCare, pp. 77-82, Springer, 2016.

[38] S. Poslad, A. Ma, Z. Wang, and H. Mei, "Using a smart city iot to incentivise and target shifts in mobility behaviour-is it a piece of pie?," Sensors, vol. 15, no. 6, pp. 13069-13096, 2015.

[39] M. Pozzi and P. Sgardelis, "Engaging self-powered environmental sensors via serious gaming," in International Conference on Applications in Electronics Pervading Industry, Environment and Society, pp. 34-40, Springer, 2016.

[40] F. Song, S. Parekh, L. Hooper, Y. K. Loke, J. Ryder, A. J. Sutton, C. Hing, C. S. Kwok, C. Pang, and I. Harvey, "Dissemination and publication of research findings: an updated review of related biases," Health Technol Assess, vol. 14, no. 8, pp. 1-193, 2010.

[41] K. Bahadoor and P. Hosein, "Application for the detection of dangerous driving and an associated gamification framework," in 2016 IEEE 4th International Conference on Future Internet of Things and Cloud Workshops (FiCloudW), pp. 276-281, IEEE, 2016. 
[42] A. Penders, J. R. Octavia, M. Caron, F. de Haan, T. Devoogdt, S. Nop, A. McAtear, O. Pieters, F. Wyffels, S. Verstockt, et al., "Solis: A smart interactive system for houseplants caring," in 2018 International Conference on Orange Technologies (ICOT), pp. 1-7, IEEE, 2018.

[43] Y. rock Zou, N. Mustafa, N. A. Memon, and M. Eid, "Eco eco: changing climate related behaviors for cellphone-based videogames," in 2015 IEEE International Symposium on Haptic, Audio and Visual Environments and Games (HAVE), pp. 1-5, IEEE, 2015.

[44] T. G. Papaioannou, N. Dimitriou, K. Vasilakis, A. Schoofs, M. Nikiforakis, F. Pursche, N. Deliyski, A. Taha, D. Kotsopoulos, C. Bardaki, et al., "An iot-based gamified approach for reducing occupants' energy wastage in public buildings," Sensors, vol. 18, no. 2 , p. $537,2018$.

[45] J. Henry, S. Tang, M. Hanneghan, and C. Carter, "A framework for the integration of serious games and the internet of things (iot)," in 2018 IEEE 6th International Conference on Serious Games and Applications for Health (SeGAH), pp. 1-8, IEEE, 2018.

[46] A. H. Kobeissi, A. Sidoti, F. Bellotti, R. Berta, and A. De Gloria, "Building a tangible serious game framework for elementary spatial and geometry concepts," in 2017 IEEE 17th International Conference on Advanced Learning Technologies (ICALT), pp. 173-177, IEEE, 2017.

[47] G. Postolache, F. Carry, F. Lourenço, D. Ferreira, R. Oliveira, P. S. Girão, and O. Postolache, "Serious games based on kinect and leap motion controller for upper limbs physical rehabilitation," in Modern Sensing Technologies, pp. 147-177, Springer, 2019.

[48] L. V. Lapão, R. Marques, J. Gregório, F. Pinheiro, P. Póvoa, and M. Mira Da Silva, "Using gamification combined with indoor location to improve nurses' hand hygiene compliance in an icu ward," in Transforming Healthcare with the Internet of Things: Proceedings of the EFMI Special Topic Conference, pp. 17-19, 2016.

[49] S. Palakvangsa-Na-Ayudhya, S. Pongchandaj, S. Kriangsakdachai, and K. Sunthornwutthikrai, "Keptaom: Savings management system to increase long term savings behavior of children," in TENCON 2017-2017 IEEE Region 10 Conference, pp. 2247-2252, IEEE, 2017.

[50] D. Caivano, F. Cassano, D. Fogli, R. Lanzilotti, and A. Piccinno, "We@ home: a gamified application for collaboratively managing a smart home," in International Symposium on Ambient Intelligence, pp. 79-86, Springer, 2017.

[51] O. Miglino, A. Di Ferdinando, R. Di Fuccio, A. Rega, and C. Ricci, "Bridging digital and physical educational games using rfid/nfc technologies," Journal of e-Learning and Knowledge Society, vol. 10, no. 3, 2014.

[52] V. Tan and S. A. Varghese, "Iot-enabled health promotion," in Proceedings of the First Workshop on IoT-enabled Healthcare and Wellness Technologies and Systems, pp. 17-18, 2016.

[53] A. Karime, H. Al-Osman, J. M. Alja'am, W. Gueaieb, and A. El Saddik, "Tele-wobble: A telerehabilitation wobble board for lower extremity therapy," IEEE Transactions on Instrumentation and Measurement, vol. 61, no. 7, pp. 1816-1824, 2012.
[54] M. Ben-Moussa, M. Rubo, C. Debracque, and W.-G Lange, "Djinni: A novel technology supported exposure therapy paradigm for sad combining virtual reality and augmented reality," Frontiers in psychiatry, vol. 8, p. 26, 2017.

[55] M. Oliveri, J. B. Hauge, F. Bellotti, R. Berta, and A. De Gloria, "Designing an iot-focused, multiplayer serious game for industry 4.0 innovation," in 2019 IEEE International Conference on Engineering, Technology and Innovation (ICE/ITMC), pp. 1-9, IEEE, 2019.

[56] Y. Wang and W. Hu, "Analysis about serious game innovation on mobile devices," in 2017 IEEE/ACIS 16th International Conference on Computer and Information Science (ICIS), pp. 627-630, IEEE, 2017.

[57] C. Ardito, P. Buono, G. Desolda, and M. Matera, "From smart objects to smart experiences: An end-user development approach," International Journal of Human-Computer Studies, vol. 114, pp. 51-68, 2018.

[58] K. Fahlquist, T. Mejtoft, and J. Karlsson, "Social media game concept within the digital zoo: new ways of connecting a tourist attraction with its visitors," in 2011 International Conference on Internet of Things and 4th International Conference on Cyber, Physical and Social Computing, pp. 170-177, IEEE, 2011.

[59] R. F. Baumeister and M. R. Leary, "The need to belong: desire for interpersonal attachments as a fundamental human motivation.," Psychological bulletin, vol. 117, no. 3, p. $497,1995$.

[60] M. Flintham, S. Benford, R. Anastasi, T. Hemmings, A. Crabtree, C. Greenhalgh, N. Tandavanitj, M. Adams, and J. Row-Farr, "Where on-line meets on the streets: experiences with mobile mixed reality games," in Proceedings of the SIGCHI conference on Human factors in computing systems, pp. 569-576, 2003. 